\title{
A REPRESENTATION OF THE INFINITESIMAL GENERATOR OF A DIFFUSION PROCESS ${ }^{1}$
}

\author{
BY L. L. HELMS
}

\author{
Communicated by J. L. Doob, May 5, 1961
}

0 . Introduction. Let $\Omega$ be a connected locally compact metric space and let $C(\Omega)$ denote, as usual, the Banach space of bounded continuous real functions on $\Omega$. A diffusion process (see [1] for definitions) is a semi-group $\left\{T_{t} ; t>0\right\}$ of positivity preserving bounded linear transformations on $C(\Omega)$ which is strongly continuous for $t>0$. Such semigroups are also required to be of local character; i.e., if $x$ vanishes in a neighborhood of a point $\xi \in \Omega$, then

$$
A x(\xi)=\lim _{t \downarrow 0} \frac{T_{t} x-x}{t}(\xi)=0 .
$$

Consider an arbitrary element $x \in C(\Omega)$. If (i) the above limit exists for all $\eta$ in a neighborhood $W$ of $\xi$, (ii) the convergence is bounded on this neighborhood, and (iii) $A x$ is continuous on $W$, then $x$ is said to be in the local domain of the operator $A$ at $\xi . x$ is said to be in the global domain, $D(A)$, of the operator $A$ whenever $W=\Omega$. Feller (see $[1]$ ) has posed the problem of characterizing the operator $A$. A local representation of such operators will be discussed in this note.

One of the essential properties of the operator $A$ is the maximum property; i.e., $A x(\xi) \leqq 0$ whenever $x$ is in the local domain of $A$ at $\xi$ and $x$ has a null maximum at $\xi$. Before discussing the representation of $A$, a few remarks concerning the denseness in $C(\Omega)$ of the global domain of the operator $A$ are in order. A null point of $A$ is a point $\xi \in \Omega$ such that $A x(\xi)=x(\xi)=0$ for all $x$ in the local domain of $A$ at $\xi$. Feller has shown that the set $N$ of null points is a closed set. He has also shown that if $x$ vanishes outside a compact set which does not meet $N$, then there is a sequence $X_{\lambda}$ in the global domain of $A$ such that $X_{\lambda} \rightarrow x$ strongly [1]. Using this result, one can show that $D(A)$ is locally dense in $C(\Omega)$ at each point $\xi \in \Omega-N$; i.e., if (i) $\xi \in \Omega-N$, (ii) $W$ is a neighborhood of $\xi$ such that $\bar{W} \subset \Omega-N$, and (iii) $x \in C(\Omega)$, then $x$ can be approximated uniformly over $\bar{W}$ by an element of $D(A)$.

Another type of point at which the operator $A$ may be degenerate is the absorption point; i.e., $\xi$ is an absorption point if there is a real

${ }^{1}$ This research was sponsored by the National Science Foundation NSF Grant 12171. 
number $c$ such that $A x(\xi)=c x(\xi)$ for all $x$ in the local domain of $A$ at $\xi$. It will be assumed throughout this note that $\xi$ is a fixed point of $\Omega$ which is neither a null point nor an absorption point. This assumption implies that there is an $x$ in the local domain of $A$ at $\xi$ such that $A x(\xi)>0 ; V$ will denote a neighborhood of $\xi$ with compact closure such that $\bar{V} \cap N=\varnothing$ and $A x>0$ on $\bar{V}$. If $\eta \in V$, then $D^{*}(A, \eta)$ will denote the functions in the local domain of $A$ at $\eta$ restricted to $\bar{V}$. $D^{*}(A)$ will denote the set of functions obtained by restricting elements of $D(A)$ to $\bar{V}$, and $C(\bar{V})$ will denote the Banach space of continuous real functions on $\bar{V}$. By the above remarks, $D^{*}(A)$ is dense in $C(\bar{V})$.

1. Generalized harmonic measures. It will be assumed in this section that $1 \in D(A)$ and $A 1=0$. Let $U$ be an open subset of $V$. The boundary of $U$ will be denoted by $U^{\prime}$. A function $y$ in $D^{*}(A, \eta)$ for all $\eta \in U$ will be called subregular (superregular) on $U$ if $A y \geqq 0$ $(A y \leqq 0)$ on $U$. A function is regular on $U$ if it is both subregular and superregular on $U$. The set $P$ of functions subregular on $U$ has the following properties:

(a) $P$ is a wedge in $C(\bar{V})$; i.e., $P$ is a convex set in $C(\bar{V})$ and $t P \subset P$ for $t \geqq 0$.

(b) $x \in P$ implies $x(\eta) \leqq \sup _{U^{\prime}} x$ for all $\eta \in U$.

(c) $P$ contains a nonzero element.

The second assertion follows from the fact that $A 1=0$ and that $A$ possesses the maximum property. These three properties suffice to prove the following theorem.

THEOREM 1. For each $\eta \in U$, there is a regular Borel measure $p(\eta, U, \cdot)$ defined on the Borel subsets of $U^{\prime}$ such that

$$
x(\eta) \leqq \int_{U^{\prime}} x(\sigma) p(\eta, U, d \sigma)
$$

for each $x$ subregular on $U$. Moreover, $p\left(\eta, U, U^{\prime}\right)=1$.

Sketch of Proof. Consider $C(\bar{V}) \times E_{1}$, the Cartesian product of $C(\bar{V})$ with the set of real numbers. The set $E$ of all pairs $(x, \alpha)$ where $x \in C(\bar{V})$ and $\alpha \geqq \sup _{U^{\prime}} x$ is a convex body in this product space. The set $F$ of all pairs $(x, x(\eta))$ where $x$ is subregular on $U$ is a convex set in the product space. Moreover, (Int $E) \cap F=\varnothing$. Using the Eidelheit separation theorem, there is a linear functional $y^{*}$ on $C(\bar{V})$ such that $x(\eta) \leqq y^{*}(x)$ for all $x$ subregular on $U$ and $y^{*}(x) \leqq \sup _{U^{\prime}} x$ for all $x \in C(\bar{V})$. The Riesz representation theorem can be used to represent the positivity preserving linear functional $y^{*}$ as a measure on $\bar{V}$. To show that this measure is concentrated on $U^{\prime}$, it need only be ob- 
served that $y^{*}(z)=0$ for any $z \in C(\bar{V})$ which is zero on $U^{\prime}$ and strictly positive elsewhere.

A measure of the type described in the preceding theorem will be called a generalized harmonic measure. The integral relative to a generalized harmonic measure will be denoted by $L(\eta, U, \cdot)$.

2. Representations. For the time being, it will be assumed that $1 \in D(A)$ and $A 1=0$. Again $U$ will be an open subset of $V$. The inequality of the following lemma is the starting point of the representation.

Lemma 2. There is an $x \in D^{*}(A)$ such that $A x>0$ on $\bar{V}, x(\eta)$ $<L(\eta, U, x)$ for all $\eta \in U$, and

$$
\inf _{\bar{U}}(A z / A x) \leqq \frac{L(\eta, U, z)-z(\eta)}{L(\eta, U, x)-x(\eta)} \leqq \sup _{\bar{U}}(A z / A x)
$$

whenever $A z$ is defined on $\bar{U}$.

SKETCH OF PROOF. One first shows that there is a $y$ (which may depend upon $\eta$ and $U$ ) such that $y(\eta)<L(\eta, U, y)$ and $A y>0$ on $\bar{U}$ as follows. Suppose the contrary; i.e., $y(\eta)=L(\eta, U, y)$ for all $y$ such that $A y>0$ on $\bar{U}$. By assumption, there is an $x$ such that $A x>0$ on $\bar{V} \supset \bar{U}$. Consider any $y$ such that $A y$ is defined on $\bar{U}$. Each such $y$ can be represented in the form $z-t x$ where $A z>0$ on $\bar{U}$ and $t$ is sufficiently large. It follows that $y(\eta)=L(\eta, U, y)$ for all $y$ for which $A y$ is defined on $\bar{U}$. But since the class of such functions is dense in $C(\bar{U})$, the evaluation linear functional $y^{*}(y)=y(\eta)$ and the linear functional $L(\eta, U, \cdot)$ are equal. This, however, is not possible. This proves that there is a $y$ such that $A y>0$ on $\bar{U}$ and $y(\eta)<L(\eta, U, y)$. A preliminary version of the lemma is now obtained as follows. Consider any $z$ such that $A z$ is defined on $\bar{U}$. For $t \geqq-\inf _{\bar{U}}(A z / A y)$, $A(z+t y) \geqq 0$ on $\bar{U}$. By Theorem $1, z(\eta)+t y(\eta) \leqq L(\eta, U, z+t y)$. Rearranging terms and letting $t$ approach $-\inf _{\bar{U}}(A z / A y)$ results in the left inequality (with $x$ replaced by $y$ ). The other inequality is proved similarly. Having proved the inequality with $x$ replaced by $y$, it follows that $z(\eta)<L(\eta, U, z)$ for any $z$ such that $A z>0$ on $\bar{V}$ and that $y$, which may depend upon $\eta$ and $U$, may be replaced by any such $z$.

In passing it is worth noting that the preceding inequality can be used to show that every generalized second order differential operator on $C(\Omega)$ as herein considered has a closed extension. The following theorem is an obvious consequence of Lemma 2. 
TheOREM 3. There is an $x \in D^{*}(A, \xi)$ such that for each $\eta \in V$ and each $z \in D^{*}(A, \eta)$

$$
A z(\eta)=A x(\eta) \lim _{U \downarrow\{\eta\}} \frac{L(\eta, U, z)-z(\eta)}{L(\eta, U, x)-x(\eta)} .
$$

The requirement that $1 \in D(A)$ and $A 1=0$ can now be removed.

THEOREM 4. There is a neighborhood $W$ of $\xi$, a function $x \in D^{*}(A, \xi)$ with $x>0$ on $\bar{W}$, and a function $y \in D^{*}(A, \xi)$ with $x A y-y A x>0$ on $\bar{W}$ such that for each $\eta \in W$ and each $z \in D^{*}(A, \eta)$

$$
\begin{aligned}
A z(\eta) & =\frac{z(\eta)}{x(\eta)} A x(\eta) \\
& +\frac{x(\eta) A y(\eta)-y(\eta) A x(\eta)}{x(\eta)} \lim _{U \downarrow\{\eta\}} \frac{L(\eta, U, z / x)-z(\eta) / x(\eta)}{L(\eta, U, y / x)-y(\eta) / x(\eta)} .
\end{aligned}
$$

Sketch of PROof. Since $D(A)$ is locally dense at $\xi$, there is an $x \in D^{*}(A, \xi)$ such that $x(\xi)>0$. Choose a neighborhood $W_{1} \subset V$ of $\xi$ such that $x>0$ on $\bar{W}_{1}$. Since $\xi$ is neither a null point nor an absorption point, there is a $y \in D^{*}(A, \xi)$ such that $x(\xi) A y(\xi)-y(\xi) A x(\xi)>0$. Choose a neighborhood $W$ of $\xi$ such that $W \subset W_{1}$ and $x A y-y A x>0$ on $\bar{W}$. After restricting all functions to $\bar{W}$, one defines an operator $B$ on quotients of the form $z / x$, where $z \in D^{*}(A, \eta)$ and $\eta \in W$, by the equation $B\left(z x^{-1}\right)=A z-z x^{-1} A x$. This operator has the essential properties used to obtain the representation of the previous theorem.

\section{REFERENCE}

1. W. Feller, The general diffusion operator and positivity preserving semi-groups in one dimension, Ann. of Math. vol. 60 (1954) pp. 417-436.

UNIVERSITY OF ILLINOIS 